\title{
Biopsy Sampling in Upper Gastrointestinal Endoscopy: A Survey from 10 Tertiary Referral Centres Across Europe
}

\author{
Jan Bornschein ${ }^{a, b, c}$ Terry Tran-Nguyen ${ }^{a, b} \quad$ Gloria Fernandez-Esparrach ${ }^{d}$ Stephen Ash $^{e}$ \\ Francesc Balaguer $^{d}$ Elizabeth L. Bird-Lieberman ${ }^{a}$ b Henry Córdova ${ }^{d}$ Zane Dzerve $^{f}$ \\ Matteo Fassan ${ }^{g}$ Marcis Leja ${ }^{f}$ Ivan Lyutakov ${ }^{h}$ Tim Middelburgi ${ }^{\text {Leticia Moreira }}{ }^{d}$ \\ Radislav Nakov $^{\text {h }}$ Stella A. V. Nieuwenburgi Anthony O'Connor ${ }^{j}$ Stefano Realdonk \\ Heiko De Schepper ${ }^{l}$ Annemieke Smet ${ }^{m}$ M. C. W. Spaander ${ }^{i}$ Ivars Tolmanis $^{f}$ Tadas Urbonas $^{n}$ \\ Jochen Weigt ${ }^{c}$ Georgina L. Hold ${ }^{\circ}$ Alexander Link ${ }^{c}$ Juozas Kupcinskas ${ }^{n}$ \\ ENIGMA: European Network for the Investigation of Gastrointestinal Mucosal Alterations

\begin{abstract}
${ }^{a}$ Translational Gastroenterology Unit, John Radcliffe Hospital, Oxford University Hospitals, Headington, Oxford,

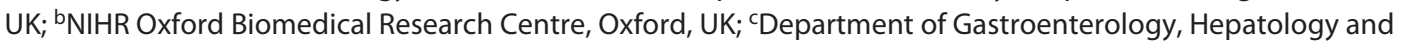
Infectious Diseases, Otto-von-Guericke University, Magdeburg, Germany; ${ }^{\mathrm{d}}$ Gastroenterology Department, Hospital Clinic of Barcelona, Institut d'Investigacions Biomèdiques August Pi i Sunyer (IDIBAPS), University of Barcelona, Centro de Investigación Biomédica en Red en Enfermedades Hepáticas y Digestivas (CIBEREHD), Barcelona, Spain; eNuffield Department of Medicine, Ludwig Institute for Cancer Research, University of Oxford, Oxford, UK; fDigestive Diseases Centre GASTRO, Institute of Clinical and Preventive Medicine, University of Latvia, Riga, Latvia;

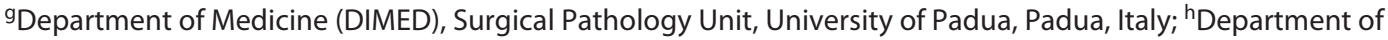
Gastroenterology, Tsaritsa Yoanna University Hospital, Medical University of Sofia, Sofia, Bulgaria; 'Department of Gastroenterology \& Hepatology, Erasmus MC University Medical Center, Rotterdam, The Netherlands; ${ }^{\text {DDepartment }}$ of Gastroenterology, Tallaght University Hospital, University of Dublin, Trinity College, Dublin, Ireland; kEndoscopy Unit, Veneto Institute of Oncology IOV-IRCCS, Padua, Italy; 'Department of Gastroenterology \& Hepatology, University Hospital Antwerp, Edegem, Belgium; 'mLaboratory of Experimental Medicine and Pediatrics, Faculty of Medicine and Health Sciences, University of Antwerp, Wilrijk, Belgium; nepartment of Gastroenterology and Institute for Digestive Research, Lithuanian University of Health Sciences, Kaunas, Lithuania; ${ }^{\circ}$ Microbiome Research Centre, St George \& Sutherland Clinical School, St George Hospital, University of New South Wales, Sydney, NSW, Australia
\end{abstract}

\section{Keywords}

Gastritis · OLGA · MAPS · Sydney · Biopsy sampling ·

Gastroscopy $\cdot$ CLO test

\section{Abstract \\ Background: Guidelines give robust recommendations on which biopsies should be taken when there is endoscopic suggestion of gastric inflammation. Adherence to these guidelines often seems arbitrary. This study aimed to give}

an overview on current practice in tertiary referral centres across Europe. Methods: Data were collected at 10 tertiary referral centres. Demographic data, the indication for each procedure, endoscopic findings, and the number and sampling site of biopsies were recorded. Findings were compared between centres, and factors influencing the decision to take biopsies were explored. Results: Biopsies were

Alexander Link and Juozas Kupcinskas equally contributed. karger@karger.com

www.karger.com/ddi

Karger $\stackrel{\text { ' }}{5}$

BOPEN ACCESS
(C) 2020 The Author(s)

Published by S. Karger AG, Basel

This is an Open Access article licensed under the Creative Commons Attribution-NonCommercial-4.0 International License (CC BY-NC) (http://www.karger.com/Services/OpenAccessLicense), applicable to the online version of the article only. Usage and distribution for commercial purposes requires written permission.
Jan Bornschein

Translational Gastroenterology Unit John Radcliffe Hospital, Headley Way Headington, Oxford OX3 9DU (UK) jan.bornschein@ouh.nhs.uk 
taken in $56.6 \%$ of 9,425 procedures, with significant variation between centres $(p<0.001)$. Gastric biopsies were taken in $43.8 \%$ of all procedures. Sampling location varied with the procedure indication $(p<0.001)$ without consistent pattern across the centres. Fewer biopsies were taken in centres which routinely applied the updated Sydney classification for gastritis assessment (46.0\%), compared to centres where this was done only upon request $(75.3 \%, p<$ $0.001)$. This was the same for centres stratifying patients according to the OLGA system $(51.8$ vs. $73.0 \%, p<0.001)$. More biopsies were taken in centres following the MAPS guidelines on stomach surveillance ( 68.1 vs. $37.1 \%, p<$ 0.001 ). Biopsy sampling was more likely in younger patients in 8 centres $(p<0.05)$, but this was not true for the whole cohort $(p=0.537)$. The percentage of procedures with biopsies correlated directly with additional costs charged in case of biopsies $(r=0.709, p=0.022)$. Conclusion: Adherence to guideline recommendations for biopsy sampling at gastroscopy was inconsistent across the participating centres. Our data suggest that centre-specific policies are applied instead.

(c) 2020 The Author(s)

Published by S. Karger AG, Basel

\section{Introduction}

Gastric cancer is a tremendous challenge for health care systems worldwide due to the high mortality associated with this disease. The reason lies in the late stage at diagnosis in most cases, with these patients experiencing no, or rather minor, non-specific symptoms in the initial phase of cancer progression. The identification of Helicobacter pylori (H. pylori) as the main risk factor and the description of a sequence of inflammation-induced preneoplastic changes of the gastric mucosa has opened the door not only to gastric cancer prevention but also to identification of individuals at increased risk of gastric cancer $[1,2]$. The latter group could be offered endoscopic surveillance to enable detection of early stage disease and thereby reduce mortality, as it has been shown for Barrett's and cancer of the oesophagus [3]. While this concept has been implemented in high incidence areas in Asia [4], this approach has not been deemed cost-effective in lowincidence countries in the West [5]. In addition to health-economic concerns, there are also biological and technical issues that render endoscopic surveillance of the stomach more challenging compared to other compartments of the luminal GI tract. Preneoplastic changes in the stomach are more subtle and more dif- ficult to identify compared to polypoid lesions in the colon. The stomach also comprises a larger surface area compared to a segment of Barrett's metaplasia in the oesophagus.

The updated Sydney protocol remains the gold standard for gastritis assessment asking for only 5 samples from the entire stomach ( 2 from the antrum, 1 from the incisura angularis, and 2 from the body), in addition to targeted biopsies from any visible abnormalities [6]. The Sydney protocol includes instructions on the histopathological grading of the inflammatory changes. Current national and international guidelines support the application of the Sydney recommendations. Countries without specific national guideline usually adopt the second edition of the European consensus on management of epithelial precancerous conditions and lesions in the stomach (MAPS II) [7-10].

Due to the biology of $H$. pylori gastritis, gastric preneoplastic conditions carry a higher risk of further progression if the gastric body is affected [11], emphasizing further the need for endoscopic surveillance in these patients [12]. Thus, all relevant risk stratification systems require distinct information on the mucosal integrity in both the gastric antrum and the body, and sampling of both areas is required to meet standards for a high quality upper gastrointestinal endoscopy [13]. The need for a complete set of biopsies was demonstrated in a large US study in 2013 analysing over 400,000 sets of biopsies [14]. Separate sampling of both the gastric antrum and the body is furthermore necessary for adequate assessment of pyloripdue $H$. pylori since a shift of the colonisation from the antrum towards the body both occurs in the natural course of the infection and under the influence of medication such as acid blockers [15].

Clinical practice shows that adherence to these recommendations for biopsy sampling is low and depends, among other factors such as local resources, on the expertise as well as personal interests of the investigator [16]. The clinical care setting also plays a role since academic centres are more likely to perform better with regards to guideline adherence [17]. Since academic centres are supposed to set the reference standards, we invited selected tertiary referral centres across Europe with high expertise in diseases of the stomach to collect data on their current practice.

The primary aim of our study was to assess standards of upper gastrointestinal biopsy sampling in patients with endoscopic signs of gastric inflammation in different academic centres across Europe and to analyse factors that might have an influence on the local practice. In addition 
to this, the sampling practice was analysed for procedures that were undertaken for different indications, such as anaemia or dysphagia, for comparison.

\section{Materials and Methods}

\section{General Study Design}

Ten of the 13 centres that were invited to join this survey of local biopsy sampling practice during upper gastrointestinal endoscopy took part in the study, including Rotterdam (The Netherlands), Antwerp (Belgium), Barcelona (Spain), Dublin (Ireland), Kaunas (Lithuania), Magdeburg (Germany), Oxford (United Kingdom), Padua (Italy), Riga (Latvia), and Sofia (Bulgaria). The centres were asked to collect data for all elective oesophagogastroduodenoscopies (OGDs) done during a period of at least 2 consecutive months and submit these in anonymised form to the lead site (Oxford). Only patients 16 years or older were included. Emergency procedures (e.g., bolus obstruction) were not included in the data set.

\section{Data Collection}

Age and sex of the patients were recorded as well as the main indication for each procedure, endoscopic findings, and the number and location of biopsies taken. A set of key indications for referral to OGD were recorded. All indications not listed in online suppl. Table 1 (for all online suppl. material, see www.karger.com/ doi/10.1159/000511867) were recorded as "other." We focused on the primary indication only in those for whom several indications were given. In 7 centres, data were recorded with the help of a onepage questionnaire that was filled out at the end of each procedure. Data were then entered into an Excel database that was submitted to the lead site. Centres using an electronic endoscopy documentation system $(n=3)$ retrieved data in a systematic way at the end of the designated study period.

We also recorded information on the cost for OGDs with and without biopsy for each centre. Centres were further asked to indicate if they followed the MAPS II guidelines on endoscopic surveillance of patients with preneoplastic conditions of the stomach. Histopathology results were not recorded as this was not the focus of the study, but each centre indicated whether histopathology scoring according to the Sydney classification and grading of changes according to the OLGA (Operative Link for Gastritis Assessment) system was done (a) as standard "routine" practice; (b) "upon request" by the endoscopist; or (c) when deemed "feasible" in case of samples with marked preneoplastic changes (or (d) not at all).

\section{Statistical Analysis}

Categorical data were compared by $\chi^{2}$ test. If not otherwise stated, $p$ values refer to the global comparison across all centres. Comparison of numerical data was done by Kruskal-Wallis test and subsequent Mann-Whitney $U$ test as post-test as appropriate. Spearman's rank correlation test was applied for correlation analyses. Test results were considered as significant for $p<0.05$. In the case of multiple comparisons, Bonferroni correction was applied resulting in different thresholds. Statistical analysis was performed using IBM SPSS Statistics for Windows, version 25 (IBM Corp., Armonk, NY, USA).

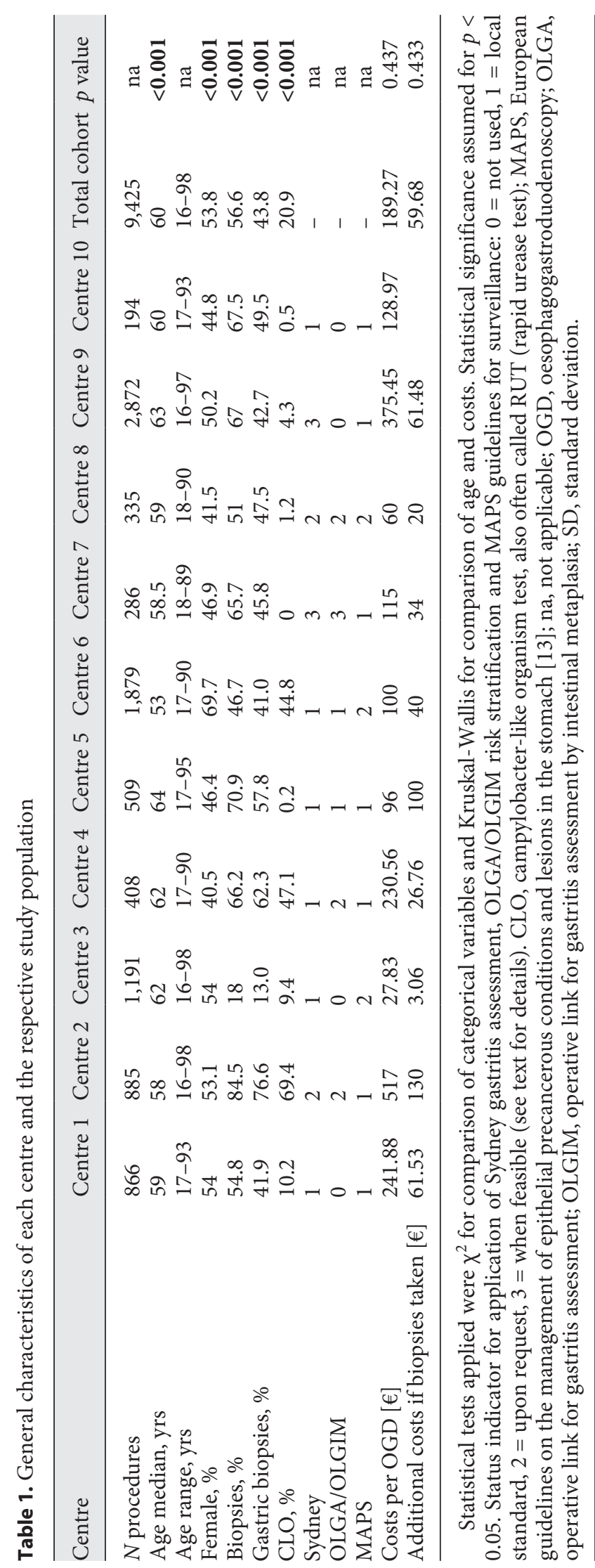


Table 2. Endoscopic findings during OGD per centre

\begin{tabular}{|c|c|c|c|c|c|c|c|c|c|c|c|}
\hline Duodenitis, \% & 6.40 & 15.00 & 3.40 & 10.00 & 4.10 & 0.50 & 1.40 & 19.40 & 6.60 & 5.20 & 6.00 \\
\hline Duodenal ulcer, \% & 2.10 & 1.40 & 5.60 & 3.40 & 1.00 & 2.10 & 1.00 & 8.10 & 1.70 & 3.10 & 2.50 \\
\hline Gastritis, \% & 22.10 & 36.70 & 22.90 & 53.90 & 39.60 & 43.50 & 9.40 & 52.80 & 22.00 & 19.60 & 30.80 \\
\hline Gastric erosions, \% & 8.00 & 1.60 & 25.00 & 12.00 & 9.60 & 7.50 & 4.20 & 11.00 & 3.80 & 5.70 & 8.40 \\
\hline PHG, \% & 6.60 & 2.10 & 1.50 & 1.00 & 4.50 & 0.10 & 1.40 & 6.90 & 3.60 & 3.60 & 2.80 \\
\hline Oesophagitis, \% & 9.60 & 21.50 & 19.60 & 13.70 & 10.60 & 15.30 & 12.60 & 35.20 & 9.50 & 19.60 & 14.50 \\
\hline Barrett's oesophagus, \% & 4.00 & 5.60 & 1.00 & 3.20 & 7.50 & 1.10 & 3.10 & 1.50 & 9.30 & 11.30 & 5.00 \\
\hline Oesophageal cancer, \% & 0.50 & 1.70 & 1.80 & 10.00 & 1.40 & 0.10 & 1.00 & 2.40 & 1.20 & 1.00 & 1.50 \\
\hline Varices, \% & 9.10 & 1.50 & 3.90 & 1.50 & 3.50 & 1.00 & 3.10 & 18.50 & 4.70 & 6.70 & 4.20 \\
\hline
\end{tabular}

OGD, oesophagogastroduodenoscopy; PHG, portal-hypertensive gastropathy.

\section{Results}

\section{General Population Characteristics per Centre}

The 10 participating centres recorded their procedures over a period of 2-4 months, covering a total of 29 operational months and 9,425 procedures between January and September 2019. There were significant differences between the centres regarding age and sex of the patient population $(p<0.001$; Table 1$)$ as well as costs per OGD $(p<0.001)$. There was also a broad variation in the additional fee that was charged if biopsies were taken during the procedure $(p<0.001$; pathology costs were not included in this consideration).

There was also variation in the indications for each procedure, as far as this was recorded ( $p<0.001$; online suppl. Table 1). About a third of patients were referred for assessment of dyspeptic symptoms. Biopsies were taken in 56.6\% of all procedures, but the sampling rate differed between centres, with the highest proportion of patients with biopsies seen in centre $2(84.5 \%)$ and the lowest in centre $3(18 \%$; Table 1, $p<0.001)$. Biopsies from the stomach were taken in $43.8 \%$ of all procedures, again with centre 2 showing the highest sampling rate $(76.6 \%)$ and centre 3 the lowest (13.0\%; Table 1, $p<0.001)$. Similarly, this also accounted for specialised biopsy-based tests such as the rapid ureasebased CLO (campylobacter-like organism) test to check for $H$. pylori, which was either used to a negligible degree or in up to $69.4 \%$ in centre 2 (Table $1, p<0.001$ ).

\section{Endoscopy Findings}

Table 2 shows the occurrence of different endoscopic findings per centre. Since we aimed to assess the rate of bi- opsy sampling in patients with inflammatory changes of the stomach, patients with signs of "gastritis" and "gastric erosions" were combined in the group "gastric inflammation." This also included patients with signs of chronic gastritis, such as endoscopic appearance of mucosal atrophy. Endoscopic scoring of atrophy was not done in all centres and was therefore not recorded for this study. Also, patients with "gastric ulcer" were not included in this group because the recommendations for biopsy sampling and endoscopic follow-up differ from non-ulcer patients. Gastric inflammation as defined above was present in $36.0 \%$ of the complete cohort with the incidence ranging from $13.6 \%$ in centre 7 to $60.5 \%$ in centre 4 ( $p<0.001$, Fig. 1a).

When gastric biopsies were taken in patients with endoscopic suggestion of gastric inflammation, these were in $24.1 \%$ pairs from antrum and body and in $26.7 \%$ sets including a sample from the incisura as required by the updated Sydney classification [6]. In 19.9\% of patients with endoscopic gastric inflammation, biopsies from duodenum, antrum, and body were obtained during the procedure. The pattern of biopsies varied considerably between centres (Fig. 1b, $p<0.001$ ). In a large proportion of these patients, either no (39.2\%) or single-location only biopsies (15.4\%) were taken from the stomach.

The proportion of procedures with biopsies taken was significantly higher in patients with gastric inflammation compared to patients without ( 65.5 vs. $51.9 \%, p<0.001$; Fig. 2a). This was also true for CLO testing ( 29.5 vs. $16.2 \%$, $p<0.001)$. Most marked in this group of patients was an increase of biopsies taken from the gastric antrum (57.6 vs. $30.5 \%$; $p<0.001$ ) and the gastric body ( 47.1 vs. $23.7 \%$; $p<0.001$, Fig. 2a). 


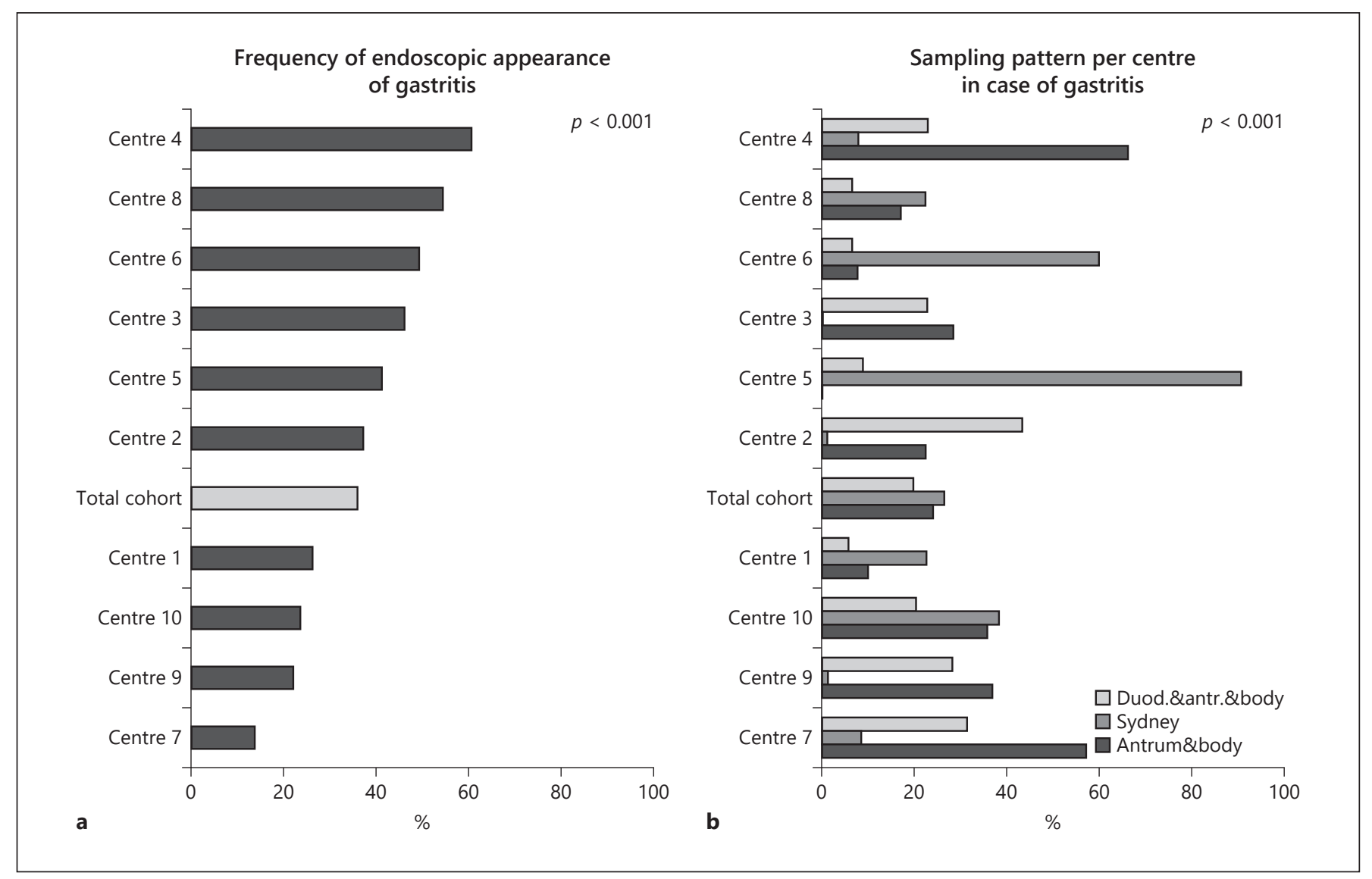

Fig. 1. Frequency of endoscopic signs of gastritis and sampling pattern. a Displayed is the prevalence of endoscopic signs of gastritis in the cohort for each centre. This was defined as mucosal irritation, for example, erythema, and presence of erosions, but did not include more advanced changes such as ulcers or tumours. b Pro- portion of sample sets taken per centre in patients with endoscopic signs of gastritis. These were mutually exclusive, but additional samples from the oesophagus might have been taken for each category that were not included in this analysis. Comparisons were done by $\chi^{2}$ test across all centres as outlined in the methods section.

\section{Influence of Indication on Biopsy Sampling}

Dyspepsia was the key indication in 2,623 cases $(27.8 \%$ of all procedures), gastro-oesophageal reflux in $813 \mathrm{pa}-$ tients $(8.6 \%)$, dysphagia in 781 procedures $(8.3 \%)$, and 887 OGDs (9.4\%) were done for investigation of anaemia (online suppl. Table 1). The sampling pattern varied significantly with indication across the total cohort $(p<$ 0.001 ; Fig. $2 \mathrm{~b}, \mathrm{c}$ ). In the individual assessment, this variation was statistically confirmed in centres $1(p=0.008)$ and $10(p=0.001)$. Duodenal samples were most frequently added to gastric sample sets for investigation of anaemia (Fig. 2b, c).

For comparison, we analysed the proportion of patients with oesophageal sampling in cases where there was an indication that would prompt biopsies, such as dysphagia (Fig. 3a) or Barrett's oesophagus (Fig. 3b). In patients investigated for dysphagia, oesophageal biopsies were taken in $44.8 \%$ of the procedures. In $13.1 \%$ of patients with dysphagia, biopsies from both antrum and body were taken in addition to multi-level oesophageal samples. Of 472 patients with endoscopic Barrett's oesophagus, $150(31.8 \%)$ underwent the OGD for endoscopic surveillance. However, data on this were incomplete (see discussion for further details). Oesophageal biopsies were taken in $71.6 \%$ of Barrett's patients compared to $10.5 \%$ in patients without this lesion $(p<0.001)$. In $31.4 \%$ of the patients with reflux symptoms, also gastric biopsies from multiple locations were taken.

\section{Other Factors Influencing Biopsy Sampling Practice}

Across the whole study population, age of subjects had no influence on the decision to take biopsies (57.9 vs. 58.1 


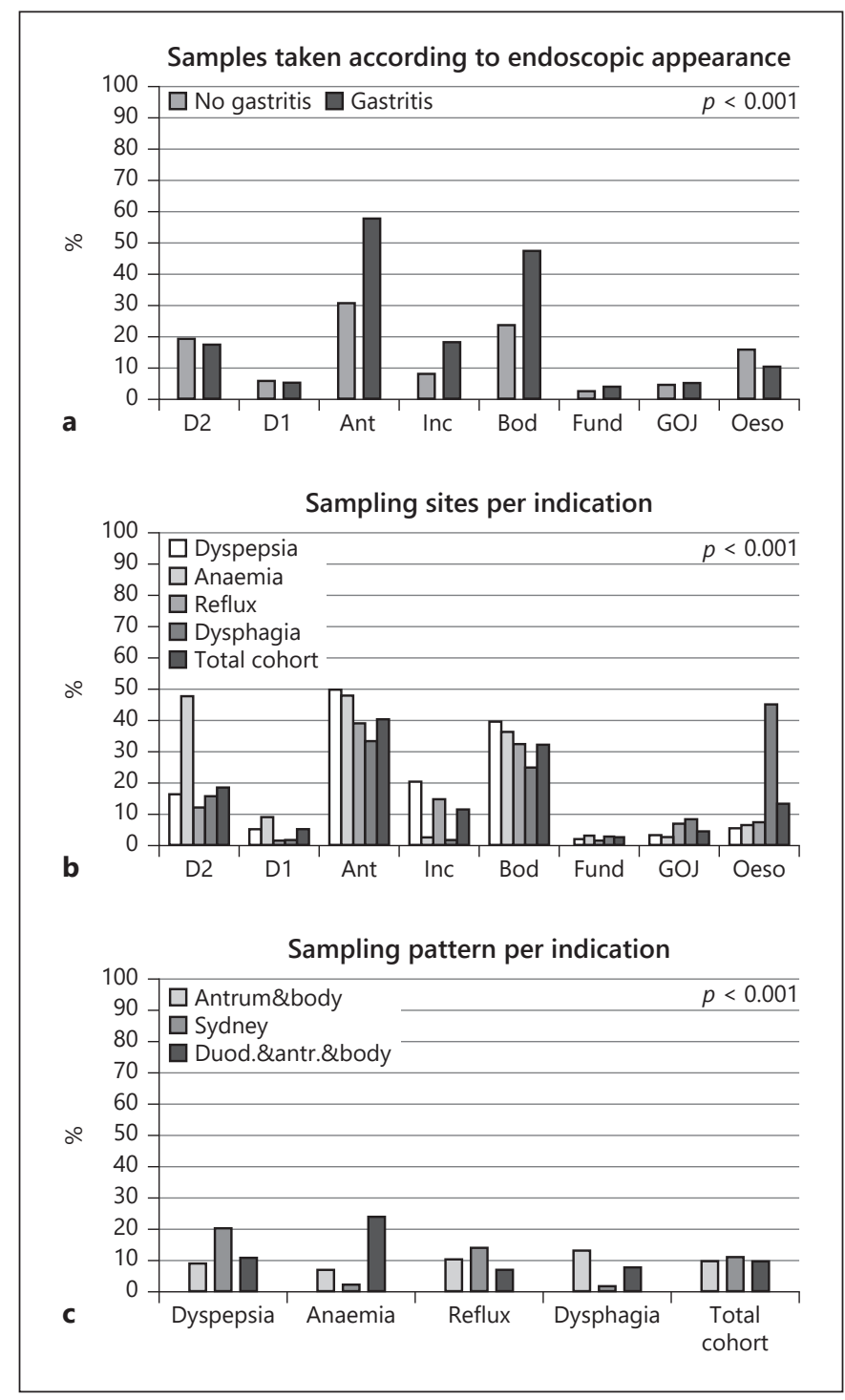

Fig. 2. Sampling pattern variation in procedures undertaken for different indication. a Comparison of the rate of samples taken from different locations in patients with and without endoscopic signs of gastritis as defined in the main text. b Proportion of biopsies taken from different sampling sites across the whole cohort according to the 4 main procedure indications. c Specific sampling patterns according to the 4 main procedure indications. Comparisons were done by $\chi^{2}$ test across all centres as outlined in the methods section. Ant, gastric antrum; Bod, gastric body; D1, duodenal bulb; D2, second part of the duodenum; Fund, gastric fundus; GOJ, gastro-oesophageal junction; Inc, Incisura; Oeso, oesophagus.

years in patients with and without biopsies, respectively; $p=0.537)$. However, when each centre was assessed individually, biopsied patients were significantly younger in 7 centres and significantly older in 1 compared to patients without sampling ( $p<0.05$; Fig. $4 a)$. There was an unexpected positive association between costs and the likelihood of biopsies, so that more biopsies were taken in case of higher per procedure costs $(r=0.709, p=0.022$; Fig. $4 \mathrm{~b})$. In line with this, the cost per OGD itself showed a similar trend ( $r=0.600, p=0.067$; data not shown).

The local practice of histopathology assessment and individual risk stratification had an influence on the sampling practice. In centres in which the updated Sydney system was routinely applied for histopathological assessment $(n=6)$, significantly fewer biopsies were taken (46.0\%) compared to the 2 centres in which Sydney scoring was done only upon specific request by the endoscopist $(75.3 \%)$ and the 2 centres in which histopathology grading was undertaken only "when feasible" in patients with marked changes $(66.8 \%$; $p<0.001$; Fig. $5 a)$. This was similar for centres which routinely applied the OLGA stratification when compared to centres where this was done "upon request" or "when feasible" (51.8 vs. 73.0 vs. $65.7 \%$; $p<0.001$; Fig. 5b). In the 2 centres in which OLGA was routinely established, sampling occurred at the same rate as in the 4 centres which did not apply OLGA at all (51.8 vs. $53.5 \%$; Fig. 5 b).

Sampling rates were significantly higher in the 7 centres which subjected their patients to endoscopic surveillance as suggested by the MAPS/MAPS II guidelines when compared to the 3 centres in which this was planned or just recently initiated (68.1 vs. $37.1 \%$; $p<0.001$; Fig. 5 c). In centres already following the MAPS surveillance recommendation more often samples were taken from both antrum and body compared to centres in which this was not yet implemented ( 34 vs. $21 \%, p<0.001$ ).

Use of anticoagulants had no significant effect on the decision whether or not to take biopsies. A detailed analysis of the subcohort from Oxford showed that only 53 patients $(1.8 \%)$ were on anticoagulant treatment which had not been stopped prior to the procedure, including 22 patients on warfarin and 31 patients on a novel oral anticoagulant. Platelet inhibition was given in 38 patients, with 30 patients being on clopidogrel, 12 on aspirin, and 4 on a combination of both. The rate of biopsy sampling was the same across all groups.

\section{Discussion}

This study delivers an overview on the current practice of gastric biopsy sampling in 10 European tertiary referral centres with a high degree of expertise in upper gastrointestinal disease. The aim was to assess adherence to na- 


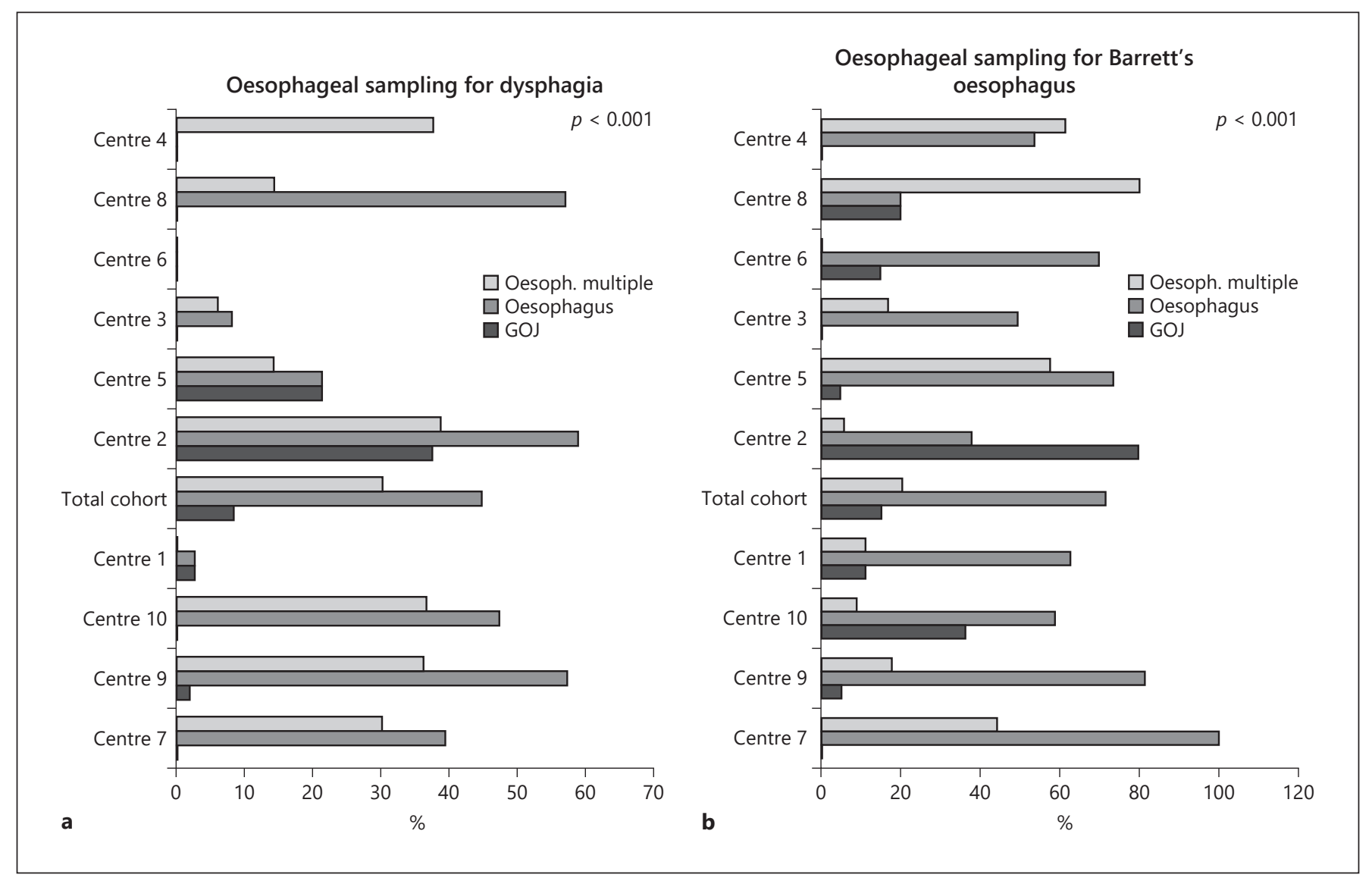

Fig. 3. Sampling of the oesophagus and the GOJ in patients with dysphagia or Barrett's oesophagus. a Sampling of GOJ and oesophagus (including multiple level sampling) in patients investigated for dysphagia. b Sampling of GOJ and oesophagus (including multiple level sampling) in patients with endoscopic signs of Barrett's

tional and international guidance and to emphasize the need for further training and education on this topic.

In our study, gastric biopsies were taken in only $60 \%$ of the patients in whom endoscopic signs of gastritis were reported (not including ulcers). Local experience in Oxford demonstrated that the rate of gastric biopsy sampling in these patients increased from 64 to $73 \%$ after a local audit undertaken prior to the study period followed by training seminars and instructive posters having been provided in endoscopy rooms. The outcome of a similar approach in Barcelona was more encouraging with a $25 \%$ increase in biopsy sampling [16]. However, the effect of training and education on the need for matching biopsies from gastric antrum and body was limited (only $4 \%$ increase) in Oxford. Both H. pylori-induced inflammation and reactive changes, due to bile reflux, often affect more the distal stomach which makes it likely that samples were

oesophagus. This included both patients under endoscopic surveillance and incidental cases. Comparisons were done by $\chi^{2}$ test across all centres as outlined in the methods section. GOJ, gastrooesophageal junction.

taken from areas with more marked erythema resulting in a higher rate of antrum samples. It is important to emphasize the need for matching body biopsies to enable complete assessment of gastric pathology [12].

The recent update of the European MAPS recommendations [12] and the inclusion of its key recommendations in the National guidelines of Germany [10] and the UK [9] put more weight on the need for an appropriate individual gastric cancer risk assessment, which aligns with recent US guidance [18]. In the current study, significantly more biopsies were taken in centres which already followed the MAPS/MAPS II surveillance recommendations. There is still debate on the most accurate risk stratification system [19]. In 2005, Rugge and Genta [20] proposed the OLGA system for staging of atrophic changes in antrum plus incisura, and body, with large, both retrospective and prospective studies supporting the feasi- 
bility and clinical relevance of this staging stratification $[21,22]$. Limitations are the interobserver variability of the pathologists scoring the mucosal changes and the need for the endoscopist to take the correct biopsies to avoid underscoring. The suggestions for overcoming the former problem was to replace the assessment of atrophy by IM only (OLGIM) [23], or combine application of OLGA plus OLGIM [24], but this has thus far found only limited acceptance.

In our study, a complete set of biopsies, as recommended by the Sydney system (including a sample from the incisura), was only taken in one out of 6 patients with endoscopic suggestion of gastric inflammation. This result is furthermore skewed by 1 centre where Sydney samples were taken in $84 \%$ of these patients, including a sample form the incisura which was reported to increase the yield for detecting preneoplastic conditions by $13 \%$ [25]. It is likely that economic factors, including time constraints during the procedures and additional costs for further biopsies, limit the willingness to obtain this additional sample with the opinions of pathologists and endoscopists slightly differing on this issue. It was a surprise that the ratio of procedures with biopsies was lower in centres in which Sydney scoring and OLGA stratification were routinely applied when compared to those in which this was not routine practice. Once again, costs might play a role here since the workload for pathologists increases substantially when these grading systems are routinely applied. It is possible that endoscopists at the respective centres take this in consideration and sample only in the case of more marked changes. Since we did not grade the endoscopic appearance of the mucosal changes, we could not fully address these issues in our study. It is of note, that despite the inclusion of centres with great expertise in gastric pathology, national and local standards varied between centres and in most departments taking part in this study endoscopic scoring of atrophic changes was not part of the clinical routine. Therefore, we did not include this factor in the lists of data to record (similarly with intestinal metaplasia) since we wanted to capture the current routine at each centre rather than creating a study setting. Future studies should include this factor, in particular, since a recent study from Porto ignited again the discussion about the actual need for gastric biopsies when appropriate endoscopic scoring of the mucosal changes is performed [26].

We did not include the histopathology costs for additional biopsies since the variables that would need to be taken into consideration (e.g., additional staining methods, routine OLGA staging, etc.) were too diverse to allow a comparison between centres in this small study.

Our study has further limitations: we did not include data on PPI intake. Samples for diagnostic assessment of H. pylori infection are usually not taken in patients on PPI. Furthermore, since the focus of this study was inflammatory changes of the stomach, some standard indi-

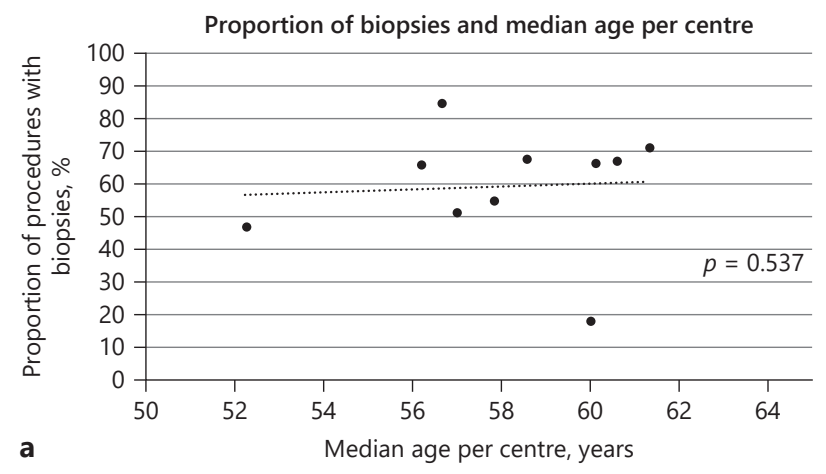

Fig. 4. Association of biopsy sampling with influencing factors. a Proportion of procedures with biopsies taken in relation to the patients' age in years as displayed on the $x$ axis. No statistically significant association was found. b Relative proportion of procedures with biopsies taken per centre in relation to additional costs for biopsy sampling in $€$ as displayed on the $x$ axis. This did not include costs for pathology, but the fixed rate added to regular fees

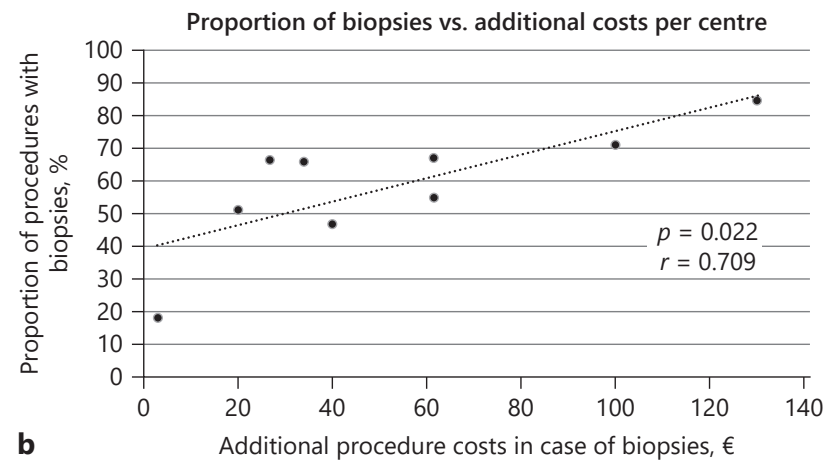

in case any biopsies were taken. Interestingly, biopsies were taken significantly more often in centres where these extra costs were higher. There was a significant association with $r=0.709, p=0.022$. Spearman's rank correlation test was applied for statistical analysis. MAPS, European guidelines on the management of epithelial precancerous conditions and lesions in the stomach; OLGA, Operative Link for Gastritis Assessment. 
cations (e.g., coeliac screening) were not included and noted under "other." When several indications were listed, we tried to stratify according to the lead indication (e.g., "dysphagia" had priority over "reflux") which might have introduced some bias. We did not systematically exclude repeat procedures (i.e., possible inclusion of patients in whom biopsies have been taken already at a previous (GD). In Oxford, the rate of repeat procedures was about $6 \%$ and did not influence the statistical outcome of the final results.

Unfortunately, we did not collect complete data on the proportion of patients undergoing the procedure for endoscopic surveillance. This included not only the indications relevant for biopsy sampling such as surveillance of atrophic gastritis and Barrett's oesophagus but also patients with portal hypertension and oesophageal varices. Although some centres provided some information on this issue, data are far from complete, as indicated by the fact that only $32 \%$ of the patients with Barrett's oesophagus were marked as "surveillance" as well as only 54 patients, that is, $0.6 \%$ of the whole cohort with gastric atrophy under surveillance. However, we believe that the influence on the main analysis of this study is minor due to these rather small numbers (compared to the 3,393 patients with endoscopic signs of gastritis).

Although oesophageal pathology was not the focus of the study, we find it encouraging that oesophageal samples were taken in $45 \%$ of patients with dysphagia compared to $14 \%$ in the total cohort, and in $72 \%$ of patients with Barrett's oesophagus compared to $11 \%$ without. Duodenal biopsies were taken in $49 \%$ of patients investigated

Fig. 5. Association of biopsy sampling with local clinical practice. a Relative proportion of procedures with biopsies taken according to local histopathology practice. Displayed are the ratios for centres which routinely score inflammatory changes routinely according to the updated Sydney protocol, those which score upon request by the endoscopist and those which score when feasible, that is, when marked changes are present. b Relative proportion of procedures with biopsies taken according to local histopathology practice. Displayed are the ratios for centres which routinely stratify patients according to the OLGA system, those which stratify upon request by the endoscopist, and those which give a staging when feasible, that is, when marked changes are present. Four centres do not apply OLGA at all. c Relative proportion of procedures with biopsies taken according to local clinical practice. Displayed are the values for centres in which surveillance regimens according to MAPS/MAPS II are fully established and for those who have planned to implement these. Comparison was done by $\chi^{2}$ test as outlined in the methods section. MAPS, European guidelines on the management of epithelial precancerous conditions and lesions in the stomach; OLGA, Operative Link for Gastritis Assessment.

Biopsy Sampling for Gastritis Assessment in Europe for anaemia compared to $17 \%$ in the total cohort. The data do not reflect the national practice for each country. It is of note that only in Germany and in the UK, evidence-based guidelines on gastritis assessment have been published $[9,10]$ and that other countries rely on international consensus recommendations $[8,12]$. An additional bias may be introduced by the healthcare infrastructure within each respective national health care system. Some countries, such as Latvia or the UK offer open access endoscopy (by direct primary care referral without specialist gastroenterology review prior to the proce-

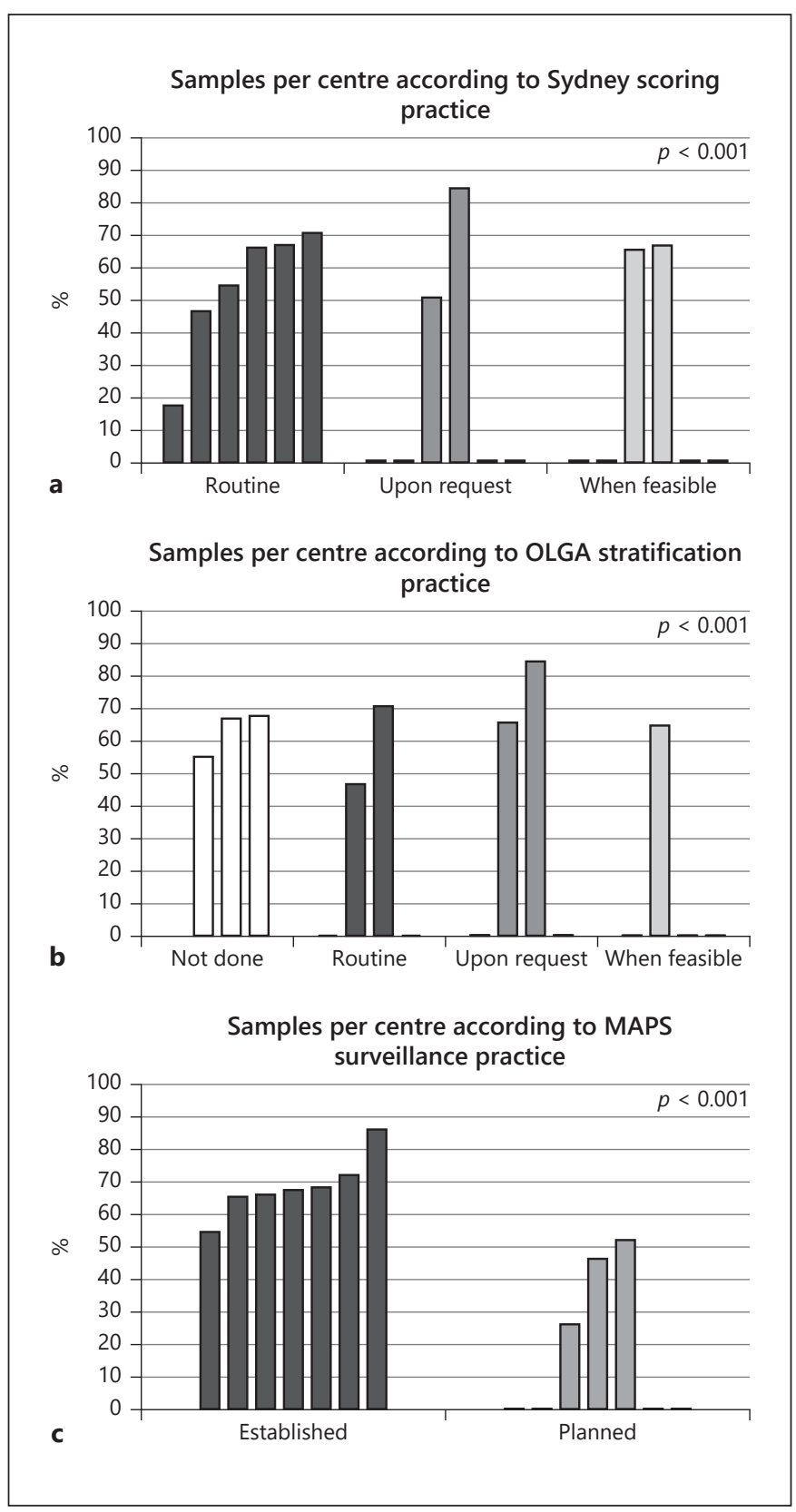


dure). This often results in referral of younger, more "healthy" patients often leading to stricter criteria as to when to take biopsies (e.g., only in patients above a certain age).

In conclusion, adherence to guideline recommendations for biopsy sampling during OGD seems to be poor even in academic centres across Europe. It has to be noted that only academic centres with high expertise in gastric pathology have been included in this study and that the data presented here cannot be directly extrapolated to conditions in community hospitals and specialist practices. A unified approach for a broader Europe-wide rollout of quality control of gastritis assessment would help to address some of the limitations of the current study and to understand the factors influencing current practice. An automated online tool facilitating a uniform database for collection of quality indicators would be ideal but setting this up is hampered by different healthcare systems and a broad variation in endoscopy documentation systems. Until this is feasible, teaching and dedicated training, which were demonstrated to be effective [16], must be supported to raise further awareness of the need for accurate assessment of inflammatory and preneoplastic changes of the stomach.

\section{Acknowledgements}

We like to thank all endoscopy staff and colleagues involved in this project for their support. We acknowledge the support of medical personnel in the Digestive Diseases Centre GASTRO, Riga, Latvia, for their support in collecting the relevant information.

\section{Statement of Ethics}

The study was locally registered as audit ID:5398 at Oxford University Hospitals, NHS Foundation Trust. Local ethics approval was sought and confirmed at each participating centre as appropriate.

\section{Conflict of Interest Statement}

S.A., F.B., E.L.B.L., J.B., H.C., Z.D., M.F., G.F.E., G.L.H., J.K., A.L., M.L., I.L., T.M., L.M., R.N., S.A.V.N., A.O'C., S.R., H.D.S., A.S., M.C.W.S., I.T., T.T.N., T.U., and J.W. have no conflict of interest with regard to the data presented here.

\section{Funding Sources}

A. Link reports grants from European Commission "Europäischer Fond für regionale Entwicklung" (EFRE), outside the submitted work. In Lithuania the work was partly supported by Lithuanian Research Council Grant no APP-2/2016. In Latvia, the methodological support was made available from the project lzp2018/1-0135. This work was also supported by the NIHR Oxford Biomedical Research Centre (The views expressed are those of the authors and not necessarily those of the NIHR or the Department of Health and Social Care). In Barcelona, we thank the CERCA Programme/Generalitat de Catalunya for the support.

\section{Author Contributions}

J.B. designed the study and prepared the main draft of this manuscript. All authors contributed to the data collection and analysis. All authors contributed to editing and revision of this manuscript, including approval of the submitted version.

\section{References}

1 Correa P. Human gastric carcinogenesis: a multistep and multifactorial process: first American cancer society award lecture on cancer epidemiology and prevention. Cancer Res. 1992;52(24):6735-40.

2 Marshall BJ, Warren JR. Unidentified curved bacilli in the stomach of patients with gastritis and peptic ulceration. Lancet. 1984;1(8390): 1311-5.

3 Ding YE, Li Y, He XK, Sun LM. Impact of Barrett's esophagus surveillance on the prognosis of esophageal adenocarcinoma: a meta-analysis. J Dig Dis. 2018;19:737-44.

4 Zhang X, Li M, Chen S, Hu J, Guo Q, Liu R, et al. Endoscopic screening in Asian countries is associated with reduced gastric cancer mortality: a meta-analysis and systematic review. Gastroenterology. 2018;155(2):347-e9.
5 Areia M, Dinis-Ribeiro M, Rocha Gonçalves F. Cost-utility analysis of endoscopic surveillance of patients with gastric premalignant conditions. Helicobacter. 2014;19(6):425-36.

6 Dixon MF, Genta RM, Yardley JH, Correa P. Classification and grading of gastritis. The updated Sydney system. International workshop on the histopathology of Gastritis, Houston 1994. Am J Surg Pathol. 1996 [cited 2018 Sep 30];20(10):1161-81.

7 Sugano K, Tack J, Kuipers EJ, Graham DY, El-Omar EM, Miura S, et al. Kyoto global consensus report on Helicobacter pylori gastritis. Gut. 2015;64(9):1353-67.

8 Malfertheiner P, Megraud F, O’Morain CA, Gisbert JP, Kuipers EJ, Axon AT, et al. Management of Helicobacter pylori infection-the maastricht V/florence consensus report. Gut. 2017;66(1):6-30.
9 Banks M, Graham D, Jansen M, Gotoda T, Coda S, di Pietro M, et al. British society of gastroenterology guidelines on the diagnosis and management of patients at risk of gastric adenocarcinoma. Gut. 2019;68(9):1545-75.

10 Moehler M, Al-Batran SE, Andus T, Arends J, Arnold D, Baretton G, et al. S3-Leitlinie Magenkarzinom-Diagnostik und Therapie der Adenokarzinome des Magens und des ösophagogastralen Übergangs. Z Gastroenterol. 2019;57:1517-632.

11 Uemura N, Okamoto S, Yamamoto S, Matsumura N, Yamaguchi S, Yamakido M, et al. Helicobacter pylori infection and the development of gastric cancer. N Engl J Med. 2001; 345(11):784-9. 
12 Pimentel-Nunes P, Libânio D, Marcos-Pinto R, Areia M, Leja M, Esposito G, et al. Management of epithelial precancerous conditions and lesions in the stomach (MAPS II): European Society of Gastrointestinal Endoscopy (ESGE), European Helicobacter and Microbiota Study Group (EHMSG), European Society of Pathology (ESP), and Sociedade Portuguesa de Endoscopia Digestiva (SPED) guideline update 2019. Endoscopy. 2019; 51(4):365-88.

13 Beg S, Ragunath K, Wyman A, Banks M, Trudgill N, Pritchard DM, et al. Quality standards in upper gastrointestinal endoscopy: a position statement of the British society of gastroenterology (BSG) and association of upper gastrointestinal surgeons of great Britain and Ireland (AUGIS). Gut. 2017;66(11): 1886-99.

14 Lash JG, Genta RM. Adherence to the Sydney system guidelines increases the detection of Helicobacter gastritis and intestinal metaplasia in 400738 sets of gastric biopsies. Aliment Pharmacol Ther. 2013;38(4):424-31.

15 Malfertheiner P, Kandulski A, Venerito M Proton-pump inhibitors: understanding the complications and risks. Nat Rev Gastroenterol Hepatol. 2017;14(12):697-710.
16 Córdova H, Sánchez-Montes C, DelgadoGuillena PG, Morales VJ, Sendino O, González-Suárez B, et al. Quality indicators for esophagogastroduodenoscopy: a comparative study of outcomes after an improvement programme in a tertiary hospital. Gastroenterol Hepatol. 2017;40(9):587-94.

17 Roumans CAM, van der Bogt RD, Steyerberg EW, Rizopoulos D, Lansdorp-Vogelaar I, Sharma P, et al. Adherence to recommendations of Barrett's esophagus surveillance guidelines: a systematic review and metaanalysis. Endoscopy. 2020;52:17-28.

18 Gupta S, Li D, El Serag HB, Davitkov P, Altayar O, Sultan S, et al. AGA clinical practice guidelines on management of gastric intestinal metaplasia. Gastroenterology. 2020; 158(3):693-702.

19 den Hollander WJ, Holster IL, den Hoed CM, Capelle LG, Tang TJ, Anten MP, et al. Surveillance of premalignant gastric lesions: a multicentre prospective cohort study from low incidence regions. Gut. 2019;68(4):585-93.

20 Rugge M, Genta RM. Staging gastritis: an international proposal. Gastroenterology. 2005; 129(5):1807-8.

21 Rugge M, Meggio A, Pravadelli C, Barbareschi M, Fassan M, Gentilini M, et al. Gastritis staging in the endoscopic follow-up for the secondary prevention of gastric cancer: a 5 -year prospective study of 1,755 patients. Gut. 2019;68(1):11-7.
22 Rugge M, Genta RM, Fassan M, Valentini E, Coati I, Guzzinati S, et al. OLGA Gastritis staging for the prediction of gastric cancer risk: a long-term follow-up study of 7,436 patients. Am J Gastroenterol. 2018;113(11): 1621-8.

23 Capelle LG, de Vries AC, Haringsma J, Ter Borg F, de Vries RA, Bruno MJ, et al. The staging of gastritis with the OLGA system by using intestinal metaplasia as an accurate alternative for atrophic gastritis. Gastrointest Endosc. 2010;71(7):1150-8.

24 Isajevs S, Liepniece-Karele I, Janciauskas D, Moisejevs G, Putnins V, Funka K, et al. Gastritis staging: interobserver agreement by applying OLGA and OLGIM systems. Virchows Arch. 2014;464(4):403-7.

25 Varbanova M, Wex T, Jechorek D, Röhl FW, Langner C, Selgrad M, et al. Impact of the angulus biopsy for the detection of gastric preneoplastic conditions and gastric cancer risk assessment. J Clin Pathol. 2016;69(1):19-25.

26 Marcos P, Brito-Gonçalves G, Libânio D, Pita I, Castro R, Sá I, et al. Endoscopic grading of gastric intestinal metaplasia on risk assessment for early gastric neoplasia: can we replace histology assessment also in the West? Gut. 2020;69(10):1762-8. 